\title{
A prospective cohort conversion study of twice-daily to once-daily extended-release tacrolimus: role of ethnicity
}

\author{
Lauren Glick¹, Fernanda Shamy², Michelle Nash², Ahmed Sokwala ${ }^{3}$ Tushar Malavade³, GV Ramesh Prasad ${ }^{4}$ \\ and Jeffrey S Zaltzman ${ }^{4^{*}}$
}

\begin{abstract}
Background: Tacrolimus is a widely used calcineurin inhibitor in kidney transplantation. It is available as twice-daily Prograf $^{\oplus}$ (Tac-BID) and once-daily Advagraf ${ }^{\oplus}$ (Tac-OD). Although therapeutically equivalent, some patients require dose adjustments to achieve similar trough concentrations $\left[C_{0}\right]$ after conversion. Tacrolimus exposure is affected by ethnicity in the de novo setting but the role of ethnicity in determining dose requirements and adjustments after conversion is unknown.
\end{abstract}

Methods: In this study, 496 renal transplant recipients (RTRs) were prospectively converted from Tac-BID to Tac-OD, with dose adjustments targeted to achieve similar $\left[C_{0}\right]$ at 12 months post-conversion. Renal function, acute rejection and Tac dose adjustments by ethnicity were analyzed.

Results: There were similar numbers of recipients from living and deceased donors. The mean transplant duration was 7 years. Of the RTRs, $60 \%$ were Caucasian and $40 \%$ were identified as belonging to an ethnic minority. There was no change in estimated renal function (eGFR) post-conversion to Tac-OD. At 12 months, 35/488 (7\%) RTRs were receiving a reduced dose, 101/488 (21\%) required a dose increase of which $77(16 \%)$ were receiving at least a 30\% increase in dose over baseline. The percentage of those in ethnic groups requiring a dose increase of $>30 \%$ varied from $8.0 \%$ for South Asians to 27.5\% for East Asians $(P=0.03)$, despite East Asians having a similar baseline dose of Tac-BID (3.59 mg/day) compared to the entire cohort (3.53 mg/day).

Conclusions: Ethnicity may play an important role in dosing requirements when converting from Tac-BID to Tac-OD, unrelated to baseline dose. Further investigation is required to determine the reasons for ethnic variability when patients are converted between tacrolimus preparations.

\section{Background}

Tacrolimus is a widely used calcineurin-inhibitor in kidney transplantation. It is available as both twice-daily Prograf (Tac-BID) and once-daily extended-release Advagraf ${ }^{\bullet}$ (Tac-OD) (Astellas Pharma Inc, Tokyo, Japan). While considered therapeutically equivalent $[1,2]$, in some patients conversion requires dose adjustments to achieve similar trough concentrations $\left[C_{0}\right]$ [3]. Our previously reported de novo experience indicated that non-Caucasians required significantly higher doses of both Tac-BID and Tac-OD to achieve equivalent serum concentrations [4]. However, in

\footnotetext{
*Correspondence: zaltzmanj@smh.ca

${ }^{4}$ Department of Medicine, Division of Nephrology, Keenan Research Institute, St. Michael's, 30 Bond St, Toronto, Ontario M5B1W8, Canada

Full list of author information is available at the end of the article
}

that study the Tac-BID and Tac-OD populations were separated by era owing to our program's protocol switch from the former to the latter. The effect of ethnicity on intra-patient dosing requirements has not been previously reported. The purpose of this prospective cohort study, therefore, was to assess if ethnicity is an important determinant of dosing requirements in a population of stable renal transplant recipients (RTRs) undergoing conversion from Tac-BID to Tac-OD.

\section{Methods}

The Greater Toronto Area has a population in excess of 5 million of which $43 \%$ identifies themselves as a visible minority and 50\% are foreign-born [5]. All stable RTRs receiving Tac-BID were converted to Tac-OD in January 
2012 due to concern about the potential for patient confusion resulting from the uncontrolled switching of tacrolimus preparations by retail pharmacists. About 500 stable RTRs, representing the entire Tac-BID population, were invited by appointment to initiate the switch. A transplant pharmacist and nurse provided the rationale for conversion and other necessary education including the need for $\left[C_{0}\right]$ monitoring at 1 and 4 weeks post-conversion. The St Michael's Research Ethics Board approved the study.

\section{Conversion}

Patients were converted from Tac-BID to Tac-OD on a $1 \mathrm{mg}: 1 \mathrm{mg}$ basis for the total daily dose unless their most recent $\left[C_{0}\right]$ on Tac-BID was $\leq 3.0 \mathrm{ng} / \mathrm{ml}$, in which case their Tac-OD dose was increased by $1 \mathrm{mg} /$ day. Blood samples to measure $\left[C_{0}\right]$ and renal function were obtained at 1 week, 4 weeks and every 3 months post-conversion. As per the protocol, the goal was to maintain similar $\left[C_{0}\right]$ levels postconversion. Changes in medication that could affect tacrolimus levels were monitored.

\section{Determination of ethnicity}

Patients were classified by ethnicity based on the patient's self-report and/or pre-transplant assessment records [6] as follows:

- Caucasian: any ancestry from Europe

- African Canadian: any ancestry from Africa either directly or via the Caribbean

- East Asian: ancestry from China, Mongolia, Japan, North or South Korea, Taiwan, Myanmar, Thailand, Laos, Cambodia, Vietnam, Malaysia, Singapore, Indonesia, or the Philippines

- South Asian: ancestry from India, Pakistan, Bangladesh, Sri Lanka, Nepal, Maldives, or Bhutan

- Middle Eastern: ancestry from Egypt, Algeria, Syria, Iraq, Iran, Lebanon, Jordan, Oman, Kuwait, Sudan, Tunisia, Morocco, Saudi Arabia, Yemen, UAE, Libya or the Palestinian Territories

- Other: used for various ethnicities present in low numbers and not otherwise included above

If a patient had mixed ancestry, the patient's self-report was used for assignment to a single category.

\section{Outcomes}

The primary outcome was to determine the frequency of RTRs who at last follow-up post-conversion, required $>30 \%$ increase in Tac-OD compared to Tac-BID to achieve a similar $\left[C_{0}\right]$. Secondary outcomes included the frequency of dose increase amongst various ethnic groups, renal function as assessed by the modification in diet renal disease equation (eGFR) and acute rejection rates post-conversion.

\section{Data and statistical analysis}

This was a prospective cohort study with patients entering at the time of conversion from January 2012 through May 2013, with analysis in July 2013 (3 to 18 months follow-up).

Patient demographics, type of transplant (living versus deceased), time since transplant and ethnicity were collected, in addition to Prograf dosage and $\left[C_{0}\right]$ at the time of conversion and Advagraf dosage and $\left[C_{0}\right]$ postconversion as previously described. The data on renal function by serum creatinine $(\mathrm{Cr})$ and eGFR were collected for 6 months pre-conversion to 18 months postconversion. However, the primary analysis of outcomes of interest was at 12 months post-conversion. Adverse events including episodes of biopsy-proven acute rejection were also recorded. The mean and standard deviation (SD) of Tac dosages were collected, and the frequency of dose increases between ethnic groups was analyzed using a chi-square test. Renal function pre- and post-conversion was compared using ANOVA.

\section{Results}

Between January 2012 and May 2013, 496 RTRs were converted from Tac-BID to Tac-OD. Of the cohort, 90\% were on prednisone and $76 \%$ were also taking either enteric-coated mycophenolic acid or mycophenolate mofetil. Post-conversion, there were no changes in medication known to interact with tacrolimus, such as diltiazem. There were no changes in any concomitant immunosuppressive agents. At the time of the last follow-up there were no allograft losses, and no episodes of acute rejection were noted. In the evaluation of renal function, eight patients were excluded from final analysis based on serum creatinine at time of conversion $>250 \mu \mathrm{mol} / \mathrm{l}$. These patients were

Table 1 Baseline demographics: $N=496$

\begin{tabular}{lc}
\hline Parameter & Value \\
\hline Age, mean (SD) & $55(12)$ \\
Male, $N(\%)$ & $303(61)$ \\
Deceased donor & 249 \\
Living donor & 247 \\
Transplant duration, years (range) & $7(0.8$ to 14) \\
Ethnicity, $N(\%)$ & $496(100)$ \\
Caucasian, $n(\%)$ & $282(56.8)$ \\
East Asian, $n(\%)$ & $91(18.3)$ \\
South Asian, $n(\%)$ & $75(15.1)$ \\
African Canadian, $n(\%)$ & $18(3.6)$ \\
Middle Eastern, $n(\%)$ & $12(2.4)$ \\
Other, $n$ (\%) & $10(2.0)$ \\
\hline
\end{tabular}




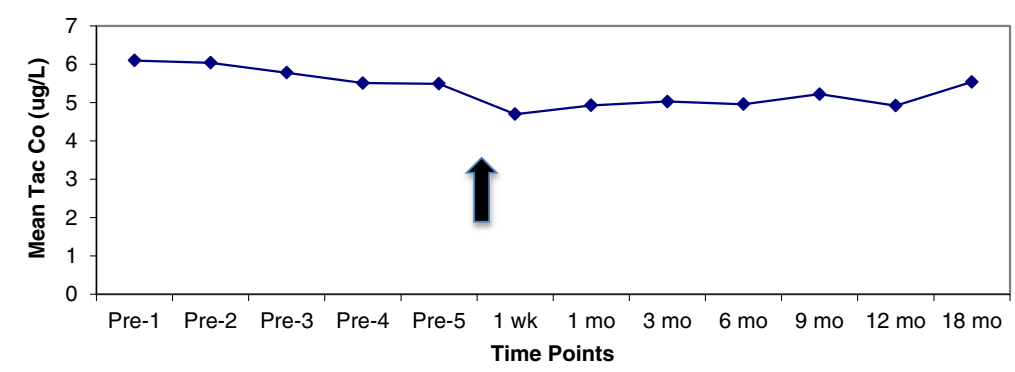

Figure 1 Mean tacrolimus trough concentration $\left[C_{0}\right](\mathrm{ng} / \mathrm{ml})$, pre- and post-conversion. Arrow indicates time of conversion.

excluded so as not to confound renal function outcomes based on conversion in patients with expected deteriorating renal function prior to conversion. Therefore, 488 patients were included in the renal function and tacrolimus dose adjustment analysis. The baseline demographics are outlined in Table 1. As shown, there were similar numbers of recipients with living and deceased donors, the mean transplant duration was 7 years, $56.8 \%$ of the RTRs were Caucasian and $40 \%$ were identified as belonging to an ethnic minority cohort.

As shown in Figure 1, at the time of conversion mean (SD) [Tac-BID] was $5.5(2.3) \mathrm{ng} / \mathrm{ml}$. At one week and one month post-conversion, [Tac-OD] decreased to $4.7(2.0)$ and $4.9(2.1) \mathrm{ng} / \mathrm{ml}$ respectively, necessitating an increased dose titration as per the protocol. By 9 months postconversion, [Tac-OD] was $5.2(2.4) \mathrm{ng} / \mathrm{ml}$. To maintain a stable [tacrolimus] there were on average 0.27 dose adjustments per patient (range: 0 to 4). Of note, by 12 months post-conversion 35/488 (7\%) RTRs were receiving a reduced dose, whereas 101/488 (21\%) required a dose increase, of which 77 (16\%) were receiving $>30 \%$ increase in dose over the baseline (Table 2). As further illustrated in Table 2, the percentage of patients requiring a dose increase of $30 \%$ or greater varied from $8.0 \%$ for South Asians to $27.5 \%$ for East Asians, the latter being statistically significant $(P=0.03)$ by chi-square analysis. We could not find any relation between initial dose and the dose increase for either the entire cohort or ethnicspecific groups. As illustrated in Figure 2, there was no change in renal function as estimated by eGFR postconversion.

\section{Discussion}

We report on the first observational cohort trial that demonstrates clear differences in tacrolimus dosages following conversion from Tac-BID to Tac-OD based on ethnicity. Almost 500 RTRs served as their own controls in this study, the second largest single-center conversion study reported to date.

The potential benefit of Tac-OD versus Tac-BID is the potential for increased patient adherence. A number of studies have described experiences with the conversion from Tac-BID to Tac-OD. The initial recommendation of conversion from Tac-BID to Tac-OD was $1 \mathrm{mg}: 1 \mathrm{mg}$, based upon pivotal studies in stable RTRs [1]. However, with increasing experience, most groups have reported that upon conversion, tacrolimus exposure, measured by either $C_{0}$ or area under the curve, is reduced in some but not all RTRs [3,7-9]. Others have explored the relation with the CYP3A5 genotype, finding dose increases upon conversion in those recipients with $C Y P 3 A 5 * 1 / * 3$ or $C Y P 3 A 5 * 1 / * 1$ compared to no increase in recipients with the CYP3A5*3/*3 [9-11]. Slatinska et al. described

Table 2 Conversion data at 12 months by ethnicity

\begin{tabular}{|c|c|c|c|c|c|c|}
\hline Conversion & $N$ & $\begin{array}{l}\text { Mean (SD) Tac-BID } \\
\text { dose (mg/day) }\end{array}$ & $\begin{array}{l}\text { Mean (SD) [Tac] } \\
\text { bid (ng/ml) }\end{array}$ & $\begin{array}{l}\text { Mean (SD) Tac-OD } \\
\text { dose (mg/day) }\end{array}$ & $\begin{array}{l}\text { Mean (SD) [Tac] } \\
\text { OD (ng/ml) }\end{array}$ & $\begin{array}{l}\text { Percentage of cohort requiring }>30 \% \\
\text { increase in Tac-OD dose }\end{array}$ \\
\hline Total & 488 & $3.53(2.01)$ & $5.49(2.36)$ & $3.75(2.03)$ & $5.15(2.48)$ & 15.7 \\
\hline Caucasian & 282 & $3.23(1.94)$ & $5.58(2.34)$ & $3.42(2.14)$ & $5.48(2.93)$ & 13.5 \\
\hline East Asian & 91 & $3.59(2.27)$ & $5.46(2.35)$ & $4.07(2.28)$ & $4.64(1.38)$ & $27.5^{*}$ \\
\hline South Asian & 75 & $3.67(2.25)$ & $5.00(1.62)$ & $3.86(2.31)$ & $4.54(1.38)$ & 8.0 \\
\hline $\begin{array}{l}\text { African } \\
\text { Canadian }\end{array}$ & 18 & 6.18 (3.87) & $5.13(1.43)$ & $6.23(4.20)$ & $5.92(2.33)$ & 11.1 \\
\hline $\begin{array}{l}\text { Middle } \\
\text { Eastern }\end{array}$ & 12 & $3.04(1.76)$ & $6.39(3.26)$ & $2.96(1.57)$ & $4.70(2.30)$ & 8.3 \\
\hline Other & 10 & $2.89(2.33)$ & $5.42(2.37)$ & $2.06(2.14)$ & $5.40(2.31)$ & 20.0 \\
\hline
\end{tabular}

$* P=0.03$ compared to all other cohorts.

$\mathrm{BID}$, twice daily; OD, once daily; SD, standard deviation; Tac, tacrolimus. 


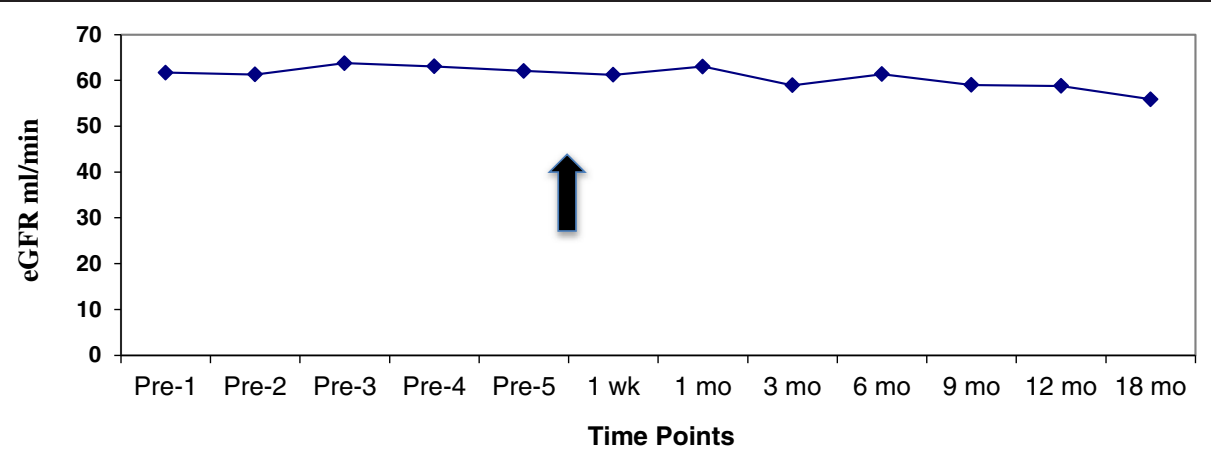

Figure 2 Mean renal function by MDRD eGRF ( $\mathrm{ml} / \mathrm{min})$, pre- and post-conversion. Arrow indicates time of conversion.

the largest single-center conversion study involving 589 RTRs. Although not reported, it is assumed by readers that the majority of these RTRs were Caucasian [12].

The renal transplant program at St Michael's in Toronto, Canada, has one of the world's most ethnically diverse populations, comprising almost 50\% new immigrants and 43\% self-identified as a visible minority. This allowed us to study prospectively dose adjustments following conversion from Tac-BID to Tac-OD, based on ethnicity. It has been well established that amongst some ethnic cohorts, such as African Americans, higher doses of tacrolimus regardless of formulation are required, because of their faster metabolism owing to a higher prevalence of CYP3A5 " $1 / * 3$ and *1/*1 and perhaps other yet unidentified polymorphisms [13]. This was also observed in our Toronto-based population with African Canadians requiring an average $6.18 \mathrm{mg} /$ day compared to $3.53 \mathrm{mg} /$ day for the entire cohort $(P=$ $0.004)$. However, only $11 \%$ of this group of 'fast metabolizers' as a whole, required an increase in Tac-OD following conversion.

Our unique observation is that upon conversion, dose increases were required with increased frequency in distinctive ethnic groups. Of almost 500 RTRs, 15.7\% required a significant dose increase to maintain equal $C_{0}$ [Tac]. However amongst RTRs of East-Asian origin, $27.5 \%$ required a significant increase in dose upon conversion from Tac-BID to Tac-OD. As stated earlier, there may be a relation between being a so-called CYP3A5 expresser $(" 1 / * 1$ or $" 1 / * 3)$ and the requirement for increased tacrolimus doses on conversion from Tac-BID to Tac-OD. However, these genotypes are not believed to be more prevalent in the East Asian population. Of interest, in a pediatric conversion study from Korea, $44 \%$ of the population required a dose increase while $26.5 \%$ required a decrease following conversion [7]. Niioka et al. reported 25\% lower 24-hr area-under-thecurve values in a conversion study involving Japanese RTRs, although this was seen mainly in those with the CYP $3 A 5 * 1 / * 3$ genotype (15\% of their cohort) [9]. Thus there may be other yet unidentified genetic polymorphisms that have a role for certain ethnic groups. Unlike the African Canadian cohort, the baseline tacrolimus dosages for the East Asian population were no different from the entire cohort $(3.59 \mathrm{mg} /$ day versus $3.53 \mathrm{mg} /$ day).

With regard to renal function, we did not find any difference in eGFR on conversion from Tac-BID to Tac-OD. Although two groups have reported an improvement in renal function following conversion $[14,15]$, this may have been due to reduced tacrolimus exposure following conversion. A multi-center conversion study of over 1,800 RTRs did not find any renal function change following conversion [16]. While there may be a renal function advantage with once daily dosing, owing to a lower single peak exposure compared with Tac-BID, more sensitive tests of GFR are likely needed to demonstrate this benefit if it exists [17].

\section{Conclusions}

The present study confirms that conversion from TacBID to Tac-OD necessitates an increase in dosage for some RTRs. The magnitude of this increase is affected by ethnicity, particularly for East Asians. Since genetic polymorphism testing is not practical for most transplant programs and unlikely to be cost effective if employed on a population-wide basis, ethnicity should be considered in determining the initial post-conversion Tac-OD dose. Nonetheless, pharmacogenetic and pharmacokinetic investigations deserve consideration to better delineate optimal conversion strategies.

\section{Abbreviations}

BID: twice daily; OD: once daily; RTR: renal transplant recipient; SD: standard deviation; Tac: tacrolimus.

\section{Competing interests}

JSZ and GVRP have previously received funding from Astellas Canada. JSZ has also received funding from Astellas Global. LG received a Keenan Summer Student Bursary from St Michael's Hospital for her work on this project. There are no other conflicts of interest to declare.

\section{Authors' contributions}

LG collected and analyzed the data. FS monitored tacrolimus levels postconversion. AS and TM collected the data. NM analyzed the data. GVRP 
managed the patients and helped to write the manuscript. JSZ was the senior author. JSZ conceived the study, analyzed the data, supervised some of the other authors and wrote the manuscript. All authors read and approved the final manuscript.

\section{Acknowledgements}

This study was in part funded by a grant from Astellas Canada.

\section{Author details}

'Keenan Summer Student Research Program, St. Michael's, 30 Bond St, Toronto, Ontario M5B21W8, Canada. ${ }^{2}$ Transplant Program, St. Michael's, 30 Bond St, Toronto, Ontario M5B1W8, Canada. ${ }^{3}$ University of Toronto Nephrology Training Program at St. Michael's, 30 Bond St, Toronto, Ontario M5B1W8, Canada. ${ }^{4}$ Department of Medicine, Division of Nephrology, Keenan Research Institute, St. Michael's, 30 Bond St, Toronto, Ontario M5B1W8, Canada.

Received: 29 November 2013 Accepted: 25 February 2014

Published: 10 March 2014

\section{References}

1. Alloway R, Steinberg S, Khalil K, Gourishanker S, Miller J, Norman D, Hariharan S, Pirsch J, Matas A, Zaltzman J, Wisemandle K, Fitzsimmons W, First MR: Two years postconversion from a Prograf-based regimen to a once-daily tacrolimus extended-release formulation in stable kidney transplant recipients. Transplantation 2007, 83:1648-1651.

2. Ho ET, Wong G, Craig JC, Chapman JR: Once-daily extended-release versus twice-daily standard-release tacrolimus in kidney transplant recipients: a systematic review. Transplantation 2013, 95:1120-1128.

3. Hougardy JM, de Jonge H, Kuypers D, Abramowicz D: The once-daily formulation of tacrolimus: a step forward in kidney transplantation? Transplantation 2012, 93:241-243.

4. Fanous H, Zheng R, Campbell C, Huang M, Nash M, Rapi L, Zaltzman JS, Prasad GVR: A comparison of the extended-release and standard-release formulations of tacrolimus in de novo kidney transplant recipients: a 12-month outcome study. Clin Kidney J 2013, 6:45-49.

5. Prasad GV, Zaltzman JS, Huang M: Renal transplantation among South Asians: a North American perspective. Transplantation 2005, 79:855.

6. Prasad GV, Vangala SK, Silver SA, Wong SC, Huang M, Rapi L, Nash MM, Zaltzman JS: South Asian ethnicity as a risk factor for major adverse cardiovascular events after renal transplantation. Clin J Am Soc Nephrol 2011, 6:204-211.

7. Min SI, Ha J, Kang HG, Ahn S, Park T, Park D, Kim SM, Hong HJ, Min SK Ha IS, Kim SJ: Conversion of twice-daily tacrolimus to once-daily tacrolimus formulation in stable pediatric kidney transplant recipients: pharmacokinetics and efficacy. Am J Transplant 2013, 13:2191-2197.

8. de Jonge $H$, Kuypers DR, Verbeke $K$, Vanrenterghem Y: Reduced Co concentrations and increased dose requirements in renal allograft recipients converted to the novel once-daily tacrolimus formulation. Transplantation 2010, 90:523-529.

9. Niioka T, Satoh S, Kagaya H, Numakura K, Inoue T, Saito M, Narita S, Tsuchiya N, Habuchi T, Miura M: Comparison of pharmacokinetics and pharmacogenetics of once-and twice-daily tacrolimus in the early stage after renal transplantation. Transplantation 2012, 94:1013-1019.

10. Glowacki F, Lionet A, Hammelin JP, Labalette M, Provot F, Hazzan M, Broly F, Noel C, Cauffiez C: Influence of cytochrome P450 3A5 (CYP3A5) genetic polymorphism on the pharmacokinetics of the prolonged-release, once-daily formulation of tacrolimus in stable renal transplant recipients. Clin Pharmacokinet 2011, 50:451-459.

11. Ro H, Min S-I, Yang J, Moon KC, Kim YS, Kim SJ, Ahn C, Ha J: Impact of tacrolimus intraindividual variability and CYP3A5 genetic polymorphism on acute rejection in kidney transplantation. Ther Drug Monit 2012, 34:680-685.

12. Slatinska J, Rohal T, Wohlfahrtova M, Viklicky O: Long-term follow-up of stable kidney transplant recipients after conversion from tacrolimus twice daily immediate release to tacrolimus once-daily prolonged release: a large single-center experience. Transplant Proc 2013, 45:1491-1496.

13. Jacobson PA, Oetting WS, Brearley AM, Leduc R, Guan W, Schladt D, Matas A Lamba V, Julian BA, Mannon RB, Israni A: Novel polymorphisms associated with tacrolimus trough concentrations: results from a multicenter kidney transplant consortium. Transplantation 2011, 91:300-308.
14. Kolonko A, Chudek J, Wiecek A: Improved kidney graft function after conversion from twice daily tacrolimus to a once daily prolonged-release formulation. Transplant Proc 2011, 43:2950-2953.

15. Tinti F, Meçule A, Poli L, Bachetoni A, Umbro I, Brunini F, Barile M, Nofroni I, Berloco PB, Mitterhofer AP: Improvement of graft function after conversion to once daily tacrolimus of stable kidney transplant patients. Transpl Proc 2010, 42:4047

16. Guirado L, Cantarell C, Franco A, Huertas EG, Fructuoso AS, Fernandez A, Gentil MA, Rodriguez A, Paul J, Torregrossa JV: Efficacy and safety of conversion from twice-daily to once-daily tacrolimus in a large cohort of stable kidney transplant recipients. Am J Transplant 2011, 11:1965-1971.

17. Zaltzman JS: A comparison of short-term exposure of once-daily extended release tacrolimus and twice-daily cyclosporine on renal function in healthy volunteers. Transplantation 2010, 90:1185-1191.

doi:10.1186/2047-1440-3-7

Cite this article as: Glick et al:: A prospective cohort conversion study of twice-daily to once-daily extended-release tacrolimus: role of ethnicity. Transplantation Research 2014 3:7.

\section{Submit your next manuscript to BioMed Central and take full advantage of:}

- Convenient online submission

- Thorough peer review

- No space constraints or color figure charges

- Immediate publication on acceptance

- Inclusion in PubMed, CAS, Scopus and Google Scholar

- Research which is freely available for redistribution 